\title{
EFEITO DOS GASES DE PROTEÇÃO NA RESISTÊNCIA À OXIDAÇÃO A ALTAS TEMPERATURAS AO AR DE JUNTAS SOLDADAS DE UM AÇO INOXIDÁVEL ATRAVÉS DO PROCESSO MIG/MAG*
}

\author{
André de Albuquerque Vicente ${ }^{1}$ \\ Débora Arruda Cabral \\ Alexandre Bueno ${ }^{3}$ \\ Hugo Hashimoto ${ }^{4}$ \\ Denise Crocce Romano Espinosa ${ }^{5}$ \\ Jorge Alberto Soares Tenório ${ }^{6}$
}

\section{Resumo}

Foram estudadas as cinéticas de oxidação a altas temperaturas ao ar de um aço inoxidável austenítico laminado e de quatro juntas soldadas produzidas através do processo MIG/MAG com diferentes gases de proteção, dentro de um projeto que visava estudar o comportamento de juntas soldadas de aços inoxidáveis austeníticos a altas temperaturas. Utilizou-se o metal base 253MA, o consumível de soldagem 22.12. HT e diferentes concentrações de gases de proteção: $100 \% \mathrm{Ar}, \mathrm{Ar}+2 \% \mathrm{CO}_{2}$, $\mathrm{Ar}+4 \% \mathrm{CO}_{2}$ e $\mathrm{Ar}+20 \% \mathrm{CO}_{2}$. Para cada mistura gasosa, foi soldado um corpo de prova. Observou-se aumento no teor de carbono e consequente diminuição da resistência à oxidação a altas temperaturas ao ar nas juntas soldadas com gases de proteção com maiores concentrações de $\mathrm{CO}_{2}$.

Palavras-chave: Aços inoxidáveis; Gases de proteção; Oxidação a altas temperaturas; MIG/MAG.

\section{EFFECT OF SHIELDING GAS IN RESISTANCE TO HIGH TEMPERATURE OXIDATION IN AIR OF WELDED JOINTS OF A STAINLESS STEEL THROUGH THE MIG-MAG PROCESS}

\begin{abstract}
The high temperature oxidation resistance in air of an austenitic stainless steel sheet and four welded joints of austenitic stainless steel produced by the MIG/MAG process with different shielding gases were studied within a project aimed the behaviour of austenitic stainless steels welded joints at high temperatures. Were used as base metal a sheet of stainless steel 253MA, the welding consumable 22.12.HT, and different protective gas concentrations: $100 \% \mathrm{Ar}, \mathrm{Ar}+2 \% \mathrm{CO}_{2}, \mathrm{Ar}+4 \% \mathrm{CO}_{2}$ and $\mathrm{Ar}+$ $20 \% \mathrm{CO}_{2}$. For each gas mixture was welded a specimen. There was an increase in carbon content and decreasing of high temperature oxidation resistance in air in weld metals deposited with shielding gases with higher concentrations of $\mathrm{CO}_{2}$.

Keywords: Stainless steels; Shielding gases; High temperature oxidation; MIG/MAG.

\footnotetext{
Doutorando do Departamento de Engenharia Química da EPUSP.

Engenheranda do Departamento de Engenharia Metalúrgica e de Materiais da EPUSP.

Mestrando do Departamento de Engenharia Química da EPUSP.

Doutorando do Departamento de Engenharia Química da EPUSP.

Professora Associada do Departamento de Engenharia Química da EPUSP.

Professor Titular do Departamento de Engenharia Química da EPUSP.
} 


\section{INTRODUÇÃO}

Quando um metal é exposto a um gás oxidante a temperatura elevada, pode ocorrer corrosão por reação direta com o gás sem a necessidade da presença de um eletrólito líquido. [1-3]

A velocidade da reação aumenta substancialmente com a temperatura. [1]

A película superficial normalmente aumenta sua espessura como resultado da reação na interface película/gás ou metal/película devido ao transporte de cátions e ânions através da película, que se comporta como um eletrólito sólido. [1-4]

Para a formação de películas contínuas e não porosas o transporte iônico através da película é a etapa controladora do processo. A estabilidade termodinâmica e certas características morfológicas da película formada são fatores chave na determinação da resistência de uma determinada liga a um meio específico. [1]

O crescimento inicial da película é normalmente bastante rápido. [1]

Se a película for sólida, não porosa e cobrir completamente a superfície do metal, a velocidade de reação irá diminuir quando a espessura da película atingir alguns milhares de angstrons conforme o transporte de massa através da camada se tornar a etapa controladora do processo. [1]

A subsequente taxa de oxidação depende dos detalhes do mecanismo de transporte, que pode ser por potencial ou gradientes de concentração ou por migração por caminhos preferenciais.

Onde a difusão for a etapa controladora do processo, a cinética normalmente segue uma lei parabólica na qual a velocidade da reação cai progressivamente com o tempo. $[1,5-14]$

A figura 1 apresenta as possíveis cinéticas de crescimento da película. [15]

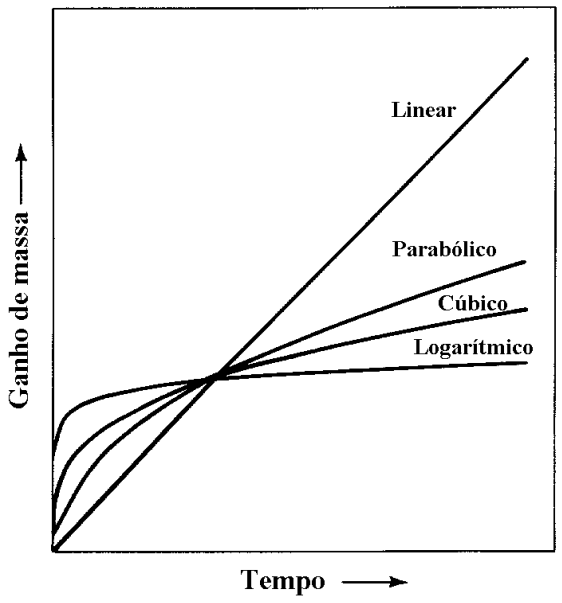

Figura 1 - Cinéticas de oxidação (ganho de massa $X$ tempo) [15]

Considere-se a reação:

$$
\mathrm{Me}+\mathrm{O}_{2}=\mathrm{MeO}_{2}
$$

A constante de equilíbrio desta reação será:

$$
K=\frac{\left[\mathrm{MeO}_{2}\right]}{[\mathrm{Me}]\left[\mathrm{O}_{2}\right]}
$$


Como: $\quad\left[\mathrm{MeO}_{2}\right]=[\mathrm{Me}]=1 \quad$ (fases distintas) $\mathrm{e}$

$\left[\mathrm{O}_{2}\right]=P_{\mathrm{O}_{2}}=$ pressão parcial de $\mathrm{O}_{2}$ em atm, resulta:

$$
K_{p}=\frac{1}{P_{O_{2}}}
$$

Pode-se demonstrar que:

$$
\Delta G^{\mathrm{O}}=-R T \ln K_{p} \quad \text { (Eq. 4) [16] }
$$

Onde: $\Delta G^{\mathrm{O}}=$ Variação de energia livre padrão da reação

A pressão parcial de oxigênio, $P_{\mathrm{O}_{2}}$, correspondente ao equilíbrio da equação 1 , chamase pressão de dissociação do óxido e ela pode ser calculada por meio da equação 3. Se num dado meio a pressão de oxigênio for $P_{O_{2}}^{\prime}$ a correspondente variação de energia livre da equação 1 será:

$$
\Delta G^{\mathrm{O}}=-R T \ln \left(\frac{P_{\mathrm{O}_{2}}^{\prime}}{P_{\mathrm{O}_{2}}}\right)
$$

Se $P_{\mathrm{O}_{2}}^{\prime}=P_{\mathrm{O}_{2}}$ então $\Delta G=0$, isto é, a reação estará em equilíbrio e tanto o metal como o óxido serão igualmente estáveis. Quando $P_{\mathrm{O}_{2}}^{\prime}<P_{\mathrm{O}_{2}}$, então $\Delta G>0$, e o óxido se torna instável, sofrendo dissociação (donde o nome para $P_{\mathrm{O}_{2}}$ ). Finalmente, para $P_{\mathrm{O}_{2}}^{\prime}>P_{\mathrm{O}_{2}}$, o valor de $\Delta G$ será negativo e o metal sofrerá oxidação espontânea. [16]

Quando vários óxidos se formam (por exemplo, $\mathrm{Fe}_{2} \mathrm{O}_{3}, \mathrm{Fe}_{3} \mathrm{O}_{4}$ e $\mathrm{FeO}$ ), os mesmos terão pressões parciais de dissociação diferentes e o óxido que for mais rico em oxigênio se dissociará antes no óxido menos rico e somente depois no metal. (16)

$\mathrm{Na}$ oxidação de ligas, a formação seletiva de um especificado óxido é governada pela reação preferencial dos elementos de liga que formam 0 composto termodinamicamente mais estável.

\section{MATERIAIS E MÉTODOS}

\subsection{Processo de soldagem e preparação dos corpos de prova soldados:}

Para a soldagem dos corpos de prova utilizou-se o processo MIG/MAG.

Foram utilizados o consumível 22.12. HT e diferentes concentrações de gases de proteção: $100 \% \mathrm{Ar}, \mathrm{Ar}+2 \% \mathrm{CO}_{2}, \mathrm{Ar}+4 \% \mathrm{CO}_{2}$ e $80 \% \mathrm{Ar}+20 \% \mathrm{CO}_{2}$. Para cada mistura gasosa, foi soldado um corpo de prova.

Ajustou-se o equipamento MIG/MAG de forma a permitir uma soldagem estável para os quatro gases de proteção.

A figura 2 descreve como os corpos de prova foram confeccionados: 

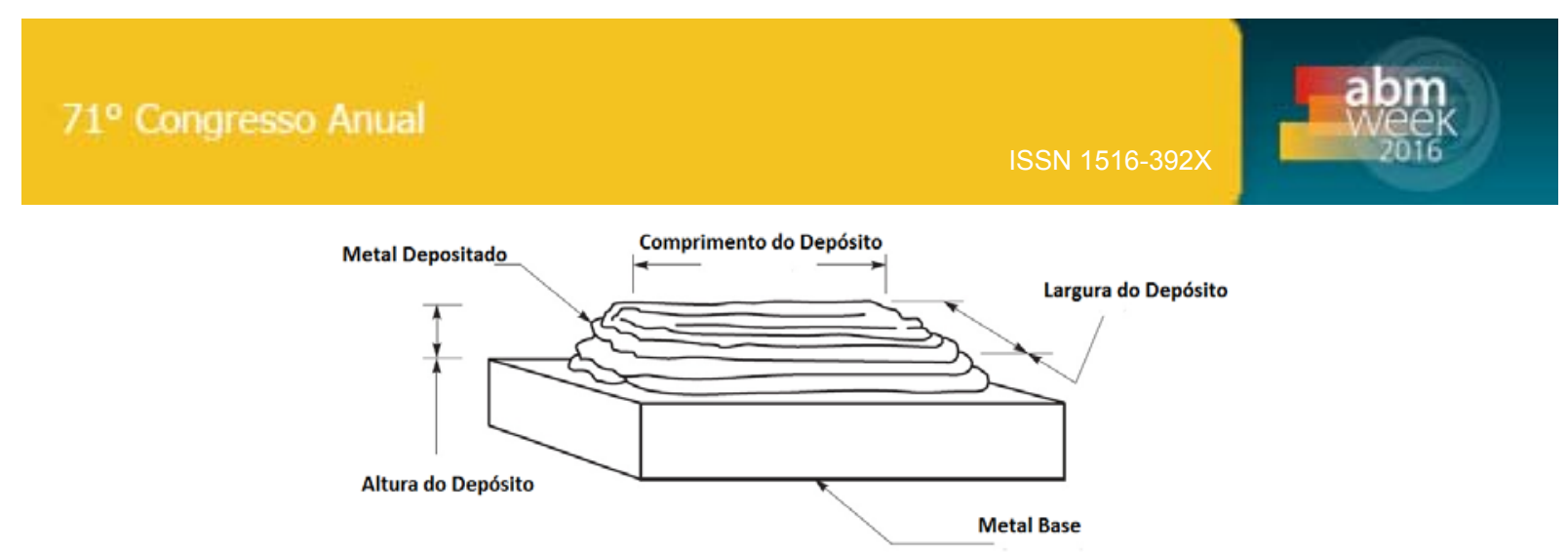

Figura 2 - Esquema da confecção dos corpos de prova soldados através de processo de soldagem MIG/MAG.

Para a confecção dos corpos de prova, utilizou-se como metal base chapas de aço inoxidável austenítico AISI 304L com 6 mm de espessura, cortadas nas dimensões 70 X $50 \mathrm{~mm}$.

O dimensional dos corpos de prova conforme a figura 2 foi:

- Comprimento do depósito:

$>50 \mathrm{~mm}$

- Altura do depósito:

$>20 \mathrm{~mm}$

- Largura do depósito:

$>30 \mathrm{~mm}$

- Quantidade de camadas depositadas:

$>5$

Utilizou-se MIG/MAG pulsado com os seguintes parâmetros de soldagem:

- Vazão de gás de proteção:

$20 \mathrm{l} / \mathrm{min}$

- Tensão:

$19-22 \mathrm{~V}$

- Corrente:

$150-200 \mathrm{~A}$

- Velocidade de soldagem:

$240-300 \mathrm{~mm} / \mathrm{min}$

A figura 3 , apresenta os corpos de prova soldados:

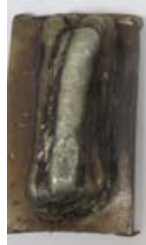

CP1

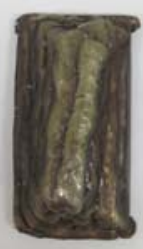

$\mathrm{CP} 2 \quad \mathrm{CP}$

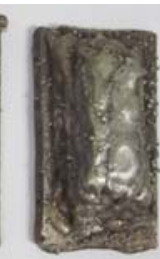

CP 4

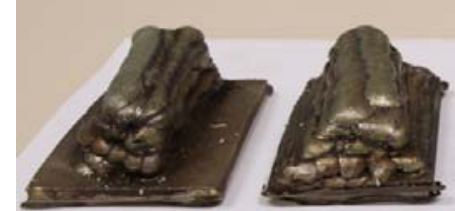

CP 2

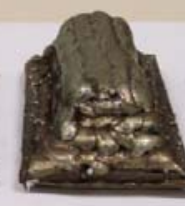

CP 3

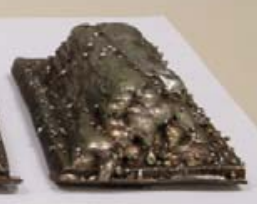

CP 4

Figura 3 - Fotos do CPs soldados. a) Vista superior b) Vista frontal.

Os gases de proteção utilizados são: CP1: 100\% Ar, CP2: $\mathrm{Ar}+2 \% \mathrm{CO}_{2}, \mathrm{CP} 3: \mathrm{Ar}+4 \% \mathrm{CO}_{2} \mathrm{e}$ CP4: $\mathrm{Ar}+20 \% \mathrm{CO}_{2}$

\subsection{Corte das amostras:}

Inicialmente, os CPs soldados foram dissecados utilizando-se um "cut-off". A figura 4 abaixo mostra um CP soldado dissecado:
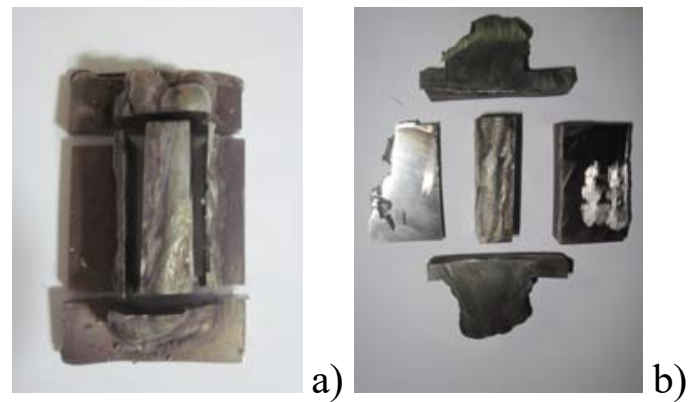

Figura 4 - Fotos de um CP soldado dissecado. Vista superior b) Vista explodida 
Para a preparação dos corpos de prova para os ensaios de oxidação ao ar a altas temperaturas objetivou-se as regiões centrais dos CPs dissecados, conforme a figura 5 abaixo.

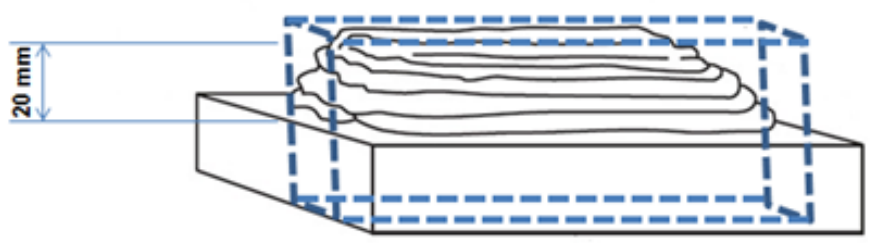

Figura 5 - Região alvo de um CP soldado para os ensaios de oxidação a altas temperaturas ao ar.

As regiões centrais dos CPs soldados dissecados, foram usinadas de forma a permitir retirar-se laminas dos metais depositados a aproximadamente $20 \mathrm{~mm}$ do metal base. Utilizando-se equipamento de corte da marca Isomet, modelo Precision Saw 1000, e disco de diamante foi possível obter corpos de prova com espessura de aproximadamente $1 \mathrm{~mm}$.

\subsection{Furação das amostras para ensaio de oxidação ao ar a altas temperaturas:}

As amostras foram furadas utilizando-se uma furadeira de bancada de marca Dremel, modelo 395 tipo 5 , e brocas de $1 \mathrm{~mm}$ de diâmetro, para poder fixa-las à haste da balança termogravimétrica.

\subsection{Polimento das amostras:}

Empregou-se o polimento manual convencional utilizando-se lixas d'água $(180,220$, 320,400 e 600 mesh) com o objetivo de se padronizar o acabamento superficial das amostras.

\subsection{Medição da área das amostras:}

As espessuras e dimensões das amostras foram determinadas com o auxílio de um micrômetro.

Através destas medições pode-se padronizar os ensaios de oxidação e obter-se os valores de ganho de massa por unidade de área.

\subsection{Limpeza das amostras:}

Antes do início dos ensaios de oxidação, todas as amostras foram limpas com álcool e, posteriormente, com acetona em aparelho de ultra-som.

Esta etapa da preparação é muito importante pois é uma forma de padronizar-se o acabamento superficial das amostras retirando resíduos de fluido de corte e/ou das lixas.

\subsection{Ensaios de oxidação:}

Os ensaios de oxidação foram realizados utilizando-se balança termogravimétrica da marca Setaram do LAT-LAREX USP. Foram realizados ensaios isotérmicos ao ar nas temperaturas $900,1000,1100^{\circ} \mathrm{C}$. 
Todos os ensaios foram realizados ao ar. O tempo de duração dos ensaios é de 24 horas.

As áreas de todas as amostras foram medidas antes do respectivo ensaio de oxidação para determinar o ganho de massa por unidade de área de forma a padronizar os ensaios.

É importante salientar que imediatamente antes do início dos ensaios de oxidação, todas as amostras foram limpas com álcool e, posteriormente, com acetona em aparelho de ultrassom.

A figura 6 apresenta o esquema de funcionamento de uma balança termogravimétrica.

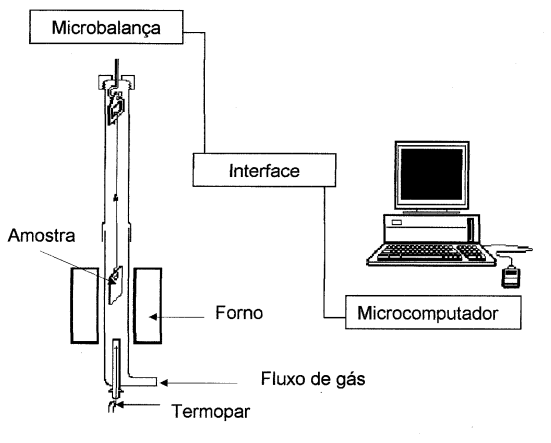

Figura 6 - Esquema de funcionamento de uma balança termogravimétrica.

\section{RESULTADOS E DISCUSSÃO}

As amostras utilizadas para os ensaios de oxidação ao ar a altas temperaturas foram obtidas através de corte de corpos de prova de um tubo de $253 \mathrm{MA} \AA$, bem como de corpos de prova soldados através do processo MIG/MAG utilizando-se diferentes gases de proteção.

Foram preparadas 4 juntas soldadas pelo processo MIG/MAG utilizando-se o consumível de soldagem 22.12.HT com diferentes gases de proteção, como segue: $100 \% \mathrm{Ar}, \mathrm{Ar}+2 \% \mathrm{CO}_{2}, \mathrm{Ar}+4 \% \mathrm{CO}_{2}$ e $80 \% \mathrm{Ar}+20 \% \mathrm{CO}_{2}$.

Desta forma, identificou-se corpos de prova soldados como CP1, CP2, CP3 e CP4 em função do gás de proteção utilizado, como segue: CP1: 100\% Ar, CP2: $\mathrm{Ar}+2 \% \mathrm{CO} 2$, CP3: $\mathrm{Ar}+4 \% \mathrm{CO}_{2}$ e CP4: $\mathrm{Ar}+20 \% \mathrm{CO}_{2}$.

Para cada mistura gasosa, foi soldado um corpo de prova.

A análise química dos corpos de prova soldados, sugerem que o aumento da concentração de $\mathrm{CO}_{2}$ nos gases de proteção aumenta o Nieq da liga através do aumento da concentração de $\mathrm{C}$ no metal depositado.

Utilizando-se as expressões propostas por DeLong para cromo e níquel equivalentes [17] é possível calcular as frações volumétricas de ferrita $\delta$ teóricas esperadas.

A tabela 1 apresenta as composições químicas do consumível utilizado e dos corpos de prova soldados, bem como os valores de cromo e níquel equivalentes utilizandose as expressões de DeLong [17] e os valores de carbono equivalente utilizando-se a expressão de O. Hammar e U. Svensson [18]. 
Tabela 1 - Composições químicas do aço laminado 253MA, do consumível de soldagem 22.12.HT e dos corpos de prova soldados

\begin{tabular}{|c|c|c|c|c|c|c|c|c|c|c|c|c|c|}
\hline & $\mathbf{C}$ & $\mathbf{S i}$ & $\mathbf{M n}$ & $\mathbf{P}$ & $\mathbf{S}$ & $\mathbf{C r}$ & $\mathbf{N i}$ & $\mathbf{N}$ & $\mathbf{C e}$ & $\mathbf{C r}_{\text {eq }}$ & $\mathbf{N i}_{\text {eq }}$ & $\mathbf{C r} / \mathbf{N i}$ & $\mathbf{C}_{\text {eq }}$ \\
\hline 253MA & 0,072 & 1,58 & 0,62 & 0,023 & 0,000 & 20,92 & 10,84 & 0,160 & 0,05 & 23,29 & 18,11 & 1,29 & 0,176 \\
\hline 22.12.HT & 0,068 & 1,58 & 0,52 & 0,016 & 0,000 & 20,90 & 10,17 & 0,170 & 0,03 & 23,27 & 17,57 & 1,32 & 0,179 \\
\hline CP1 & 0,059 & 1,51 & 0,50 & 0,016 & 0,000 & 20,12 & 10,31 & 0,172 & 0,03 & 22,39 & 17,49 & 1,28 & 0,171 \\
\hline CP2 & 0,069 & 1,45 & 0,48 & 0,016 & 0,000 & 20,10 & 10,33 & 0,166 & 0,03 & 22,28 & 17,62 & 1,26 & 0,177 \\
\hline CP3 & 0,072 & 1,38 & 0,46 & 0,016 & 0,000 & 20,10 & 10,32 & 0,162 & 0,03 & 22,17 & 17,57 & 1,26 & 0,177 \\
\hline CP4 & 0,093 & 1,36 & 0,45 & 0,016 & 0,000 & 20,04 & 10,33 & 0,162 & 0,03 & 22,08 & 18,21 & 1,21 & 0,198 \\
\hline
\end{tabular}

Creq $=\% \mathrm{Cr}+\% \mathrm{Mo}+1,5 \% \mathrm{Si}+0,5 \% \mathrm{Nb}$ e $\mathrm{Nieq}=\% \mathrm{Ni}+0,5 \% \mathrm{Mn}+30 \% \mathrm{C}+30 \% \mathrm{~N}$ [17]

$\mathrm{Ceq}=\% \mathrm{C}+0,65 \% \mathrm{~N}[18]$

A figura 7 apresenta as curvas de ganho de massa em função do tempo, obtidas para a amostra do aço inoxidável austenítico laminado (253 MA®) e para os 4 corpos de prova soldados, nos ensaios isotérmicos de oxidação ao ar por 24 horas nas temperaturas 900,1000 e $1100^{\circ} \mathrm{C}$.
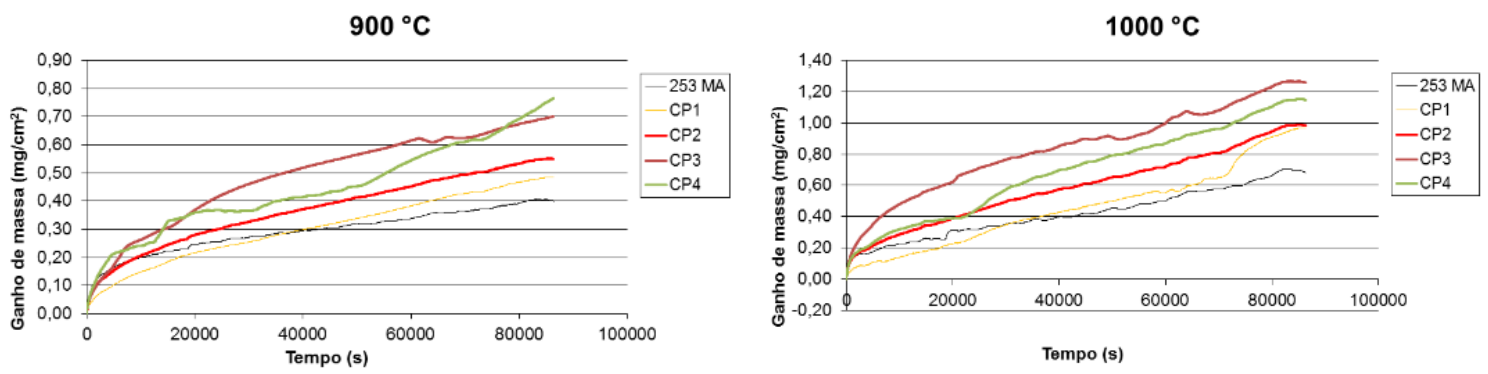

$1100^{\circ} \mathrm{C}$

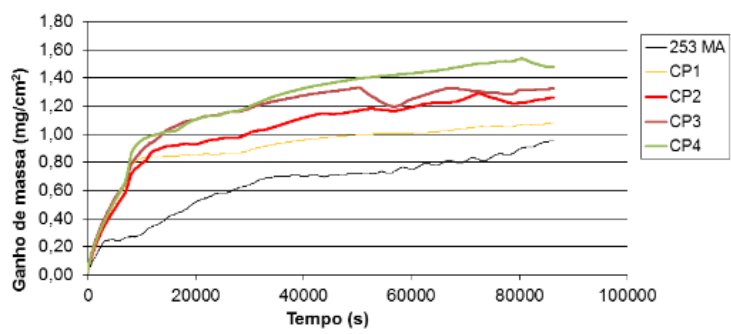

Figura 7 - Ensaios de oxidação ao ar por 24 horas a 900, 1000 e $1100{ }^{\circ} \mathrm{C}$.

Na figura 7 observa-se, para as 5 ligas estudadas, o aumento das cinéticas de oxidação com o aumento da temperatura de ensaio de 900 até $1100^{\circ} \mathrm{C}$.

Foram testados os comportamentos parabólico, exponencial e linear para as cinéticas de crescimento dos óxidos formados.

A análise dos ensaios termogravimétricos do aço inoxidável austenítico laminado (253 $\mathrm{MA} \otimes)$ bem como das 4 juntas soldadas, mostrou uma cinética de oxidação a altas temperaturas complexa, passando de logarítmica nos instantes iniciais para um posterior comportamento parabólico.

$\mathrm{O}$ aumento da concentração de $\mathrm{CO}_{2}$ nos gases de proteção resulta em juntas soldadas menos resistentes à corrosão a altas temperaturas quando comparadas com o metal base 253MA.

\section{CONCLUSÃO}

$\mathrm{O}$ aumento da concentração de $\mathrm{CO}_{2}$ nos gases de proteção aumenta o teor de $\mathrm{C}$ no metal depositado através do processo MIG/MAG. 
As juntas soldadas apresentam diminuições significativas nos teores de $\mathrm{Cr}$, $\mathrm{Ni}$, Si e Mn em relação à composição química do consumível de soldagem. O aumento da concentração de $\mathrm{CO}_{2}$ nos gases de proteção acentua estas perdas.

$\mathrm{O}$ aumento da concentração de $\mathrm{CO}_{2}$ nos gases de proteção resulta em juntas soldadas menos resistentes à corrosão a altas temperaturas quando comparadas com o metal base 253MA devido à diminuição do Creq

Os resultados obtidos sugerem que com o aumento da concentração de $\mathrm{CO}_{2}$ nos gases de proteção ocorra oxidação seletiva dos elementos de liga $\mathrm{Cr}, \mathrm{Ni}$, Si e $\mathrm{Mn}$ durante a deposição dos metais de solda através do processo MAG.

\section{Agradecimentos}

Ao Prof. Manuel Saraiva Clara pelo apoio incondicional na elaboração da parte prática deste trabalho permitindo a utilização de todos os recursos da Soudage - Welding Experts, além das inúmeras discussões técnicas.

À SMT - Sandvik Materials Technology, representada pelo caro colega politécnico Fausto Camargo e pelo caro amigo Luiz Gobbis, por acreditar neste projeto e pela doação do metal base e do consumível de soldagem bem como por sempre apoiar a Escola Politécnica da Universidade de São Paulo quando existe o interesse em realizar-se estudos envolvendo ligas resistentes à corrosão.

À Prax Air representada pelos caros colegas Marcos Lobato e Jeferson Costa, por acreditar neste projeto e pela doação dos gases de proteção para a soldagem e do ar sintético para os ensaios de oxidação bem como por sempre apoiar a Escola Politécnica da Universidade de São Paulo quando existe o interesse em realizar-se estudos envolvendo gases especiais.

\section{REFERÊNCIAS}

1. WRIGTH, I. G. High-Temperature Corrosion, Metals Handbook, 9. ed., v. 13, p. 97-103.

2. BIRKS, N. ; MEIER, G. H. Mechanisms of Oxidation: Introduction to High Temperature Oxidation of Metals, p.31-65, 1983.

3. BETTERIDGE, W.. Environments, Corrosion - Metal / Environment Reactions, chapter 7, High Temperature Corrosion, v. 1, p. 7:3-7:12, 1979.

4. BETTERIDGE, W.. Environments, Corrosion - Metal / Environment Reactions, chapter 7, High Temperature Corrosion, 3. Ed., vol. 1, p. 7:3-7:15, 1995.

5. TENÓRIO, J. A. S.; ESPINOSA, D. C. R.; GROTH, J. L.; REBELLO, M. C. "Oxidação a Alta Temperatura de Ligas à base de Ni", $2^{\circ}$ Congresso Internacional de Tecnologia Metalúrgica e de Materiais. São Paulo, 1997;

6. BARRET, C. A. The Effect of Variations of Cobalt Content on the Cyclic Oxidation Resistance of Selected Ni-Base Superalloys, Alternate Alloying for Environmental Resistance, Proceedings of the Symposium sponsored by the Corrosion and Environmental Effects Committee of The Metallurgical Society of AIME, Louisiana, $p$. 211-231, 1986.

7. SANTORELLI, R.; NORTON, J. F.; BREGANI, F. High Temperature Corrosion of Some $\mathrm{Fe}-\mathrm{Ni}-\mathrm{Cr}$ Commercial Alloys in Gaseous Environments with Relevance to Coal Conversion Processes, 11th International Corrosion Congress. Florence, v.4, p. 4.1-4.10 1990;

8. DOYCHAK, J. K.; MITCHELL, T. E; SMIALEK, J. L. High Temperature Oxidation of $\beta$ $\mathrm{NiAl}$, High-Temperature Ordered Intermetallic Alloys, Materials Research Society Symposium Proceedings, v. 39, p. 475-484, 1984. 
9. BARNES, J. J.; SRIVASTAVA, S. K. Oxidation of Gas Turbine Seal Ring Alloys and Its Effect on Mechanical Properties. 7th International Symposium on Superalloys. TMS, ASM, ASME, p. 825-833, 1992.

10. BRILL, U. ; KLÖWER, J. High Temperature Corrosion of Intermetallic Phases Based on Ni3Al. High-Temperature Ordered Intermetallic Alloys IV, Materials Research Society Symposium Proceedings, v. 213, p. 963-968, 1990.

11. PINT, B. A.; JAIN, A.; HOBBS, L. W. The Effect of Yttrium Ion Implantation on the High Temperature Oxidation Properties of NiAl, High-Temperature Ordered Intermetallic Alloys IV, Materials Research Society Symposium Proceedings, v. 213, p. 981-986, 1990.

12. WOLYNEC, S. Oxidação de Metais, Corrosão e Proteção Contra Corrosão de Metais. IPT 1981. (Publicação IPT N 1127), Coordenador Deniol K. Tanaka, p. 75-104.

13. NARDOU, F.; RANAIVONIARIVO, L.; RAYNAUD, P.; BILLY M. Relaxation of the Mechanical Stresses Developed Through Oxide Scales During Oxidation of Metals, High Temperature Alloys-Their Exploitable Potential, Commission of The European Communities, p. 89-96.

14. MCKEE, D. W.; FLEISCHER, R. L. Oxidation Behavior of Advanced Intermetallic Compounds, High-Temperature Ordered Intermetallic Alloys IV, Materials Research Society Symposium Proceedings, v. 213, 1990, p. 969-974.

15. R.D. Kane, High-Temperature Gaseous Corrosion, Corrosion: Fundamentals, Testing, and Protection, Vol.13A, ASM Handbook, ASM International, 2003, p 228-235.

16. WASIELEWSKI, G. E. ; RAPP, R. A. High-Temperature Oxidation, The Superalloys, Vital High Temperature Gas Turbine Materials for Aerospace and Industrial Power, p. 287316, 1972.

17. DeLONG, W. T.. A modified phase diagram for stainless steel weld metals. Metal Progress, p. 98-100B, 1960.

18. HAMMAR, O.; SVENSSON, U.. Influence of Steel Composition on Segregation and Microstructure During Solidification of Austenitic Stainless Steels. Solidification and Casting Metals, London, Metals Society, p. 401- 410, 1979. 